\title{
Effects of exercise training in patients with idiopathic pulmonary arterial hypertension
}

\author{
F.S. de Man*, M.L. Handoko*,\#, H. Groepenhoff*, A.J. van 't Hul", J. Abbink', \\ R.J.H. Koppers ${ }^{\S}$, H.P. Grotjohan ${ }^{f}$, J.W.R. Twisk**, H-J. Bogaard*, A. Boonstra*, \\ P.E. Postmus*, N. Westerhof*,\#, W.J. van der Laarse ${ }^{\#}$ and A. Vonk-Noordegraaf*
}

ABSTRACT: We determined the physiological effects of exercise training on exercise capacity and quadriceps muscle function in patients with idiopathic pulmonary arterial hypertension (iPAH).

In total, 19 clinically stable iPAH patients (New York Heart Association II-III) underwent a supervised exercise training programme for the duration of 12 weeks. Maximal capacity, endurance capacity and quadriceps function were assessed at baseline and after 12 weeks. In 12 patients, serial quadriceps muscle biopsies were obtained.

6-min walk distance and peak exercise capacity did not change after training. However, endurance capacity improved significantly after training, demonstrated by a shift of the anaerobic threshold to a higher workload (from $32 \pm 5$ to $46 \pm 6 \mathrm{~W} ; p=0.003$ ) together with an increase in exercise endurance time $(p<0.001)$. Moreover, exercise training increased quadriceps strength by $13 \%(p=0.005)$ and quadriceps endurance by $34 \%(p=0.001)$.

Training enhanced aerobic capacity of the quadriceps, by increasing capillarisation $(1.36 \pm 0.10$ to $1.78 \pm 0.13$ capillaries per muscle fibre; $p<0.001$ ) and oxidative enzyme activity, especially of the type-I (slow) muscle fibres. No changes were found in cross-sectional area and fibre type distribution.

Exercise training in IPAH improves exercise endurance and quadriceps muscle function, which is also reflected by structural changes of the quadriceps.

KEYWORDS: Biopsy, exercise training, pathophysiology, pulmonary hypertension, skeletal muscle dysfunction

1 diopathic pulmonary arterial hypertension (iPAH) is a life threatening disease, which eventually leads to right heart failure. A high pulmonary vascular resistance and right ventricular dysfunction impair stroke volume, thereby limiting oxygen supply to the skeletal muscles, especially during exercise, resulting in lactic acidosis at low work rates and impaired functional capacity $[1,2]$.

Traditionally, exercise training in iPAH patients was contraindicated due to the risk of sudden cardiac death [3]. However, with the increase in medical treatment options in the last decennium, the prognosis has improved significantly, and the role of exercise training in patients with $\mathrm{PAH}$ was reconsidered [3]. Recently, the first clinical trial on exercise training in patients with pulmonary arterial hypertension reported promising results of improved exercise capacity and quality of life [4].
Exercise training is a well-established adjunct therapy in several chronic diseases such as chronic obstructive pulmonary disease (COPD) and congestive heart failure $[5,6]$. In patients with these chronic diseases, skeletal muscle dysfunction contributes to exercise intolerance [7-9]. The beneficial effects of exercise training in COPD and congestive heart failure are partially attributed to improved skeletal muscle efficiency $[10,11]$ and increased capillary density and oxidative enzyme activity in quadriceps muscle biopsies [11-13].

Also in iPAH patients, skeletal muscle dysfunction has been reported. Respiratory muscle dysfunction was found in two studies, both by voluntary and nonvoluntary techniques [14, 15]. More recently, forearm muscle dysfunction has been reported in these patients [16]. In a pilot study, examining voluntary strength of the

Earn CME accreditation by answering questions about this article. You will find these at the back of the printed copy of this issue or online at www.erj.ersjournals.com/current.dtl

AFFILIATIONS

Depts of *Pulmonology, and

\#Physiology, Institute for

Cardiovascular Research, and

**Dept of Clinical Epidemiology and

Biostatistics, The Institute for

Research in Extramural Medicine, VU

University Medical Center,

Amsterdam,

"Breda Rehabilitation Center

Foundation, Breda,

+Rijnlands Rehabilitation Center,

Leiden,

${ }^{\S}$ Dept of Pulmonology, Leeuwarden

Medical Center, Leeuwarden, and

${ }^{f}$ Dept of Pulmonology, Isala Clinics,

Zwolle, The Netherlands.

CORRESPONDENCE

A. Vonk Noordegraaf

Dept of Pulmonology

VU University Medical Center

Boelelaan 1117

1081 HV Amsterdam

The Netherlands

E-mail: a.vonk@vumc.nl

Received:

Feb 172009

Accepted after revision:

March 132009 
respiratory, forearm and quadriceps muscles in iPAH patients, specific quadriceps muscle dysfunction has been found [17]. However, these studies focused on muscle function only, without giving more insight in the roles of muscle atrophy, fibre type switching, decreased oxidative enzyme activity, or reduced capillary density, as potential underlying mechanisms of skeletal muscle dysfunction [18].

We hypothesised that exercise training in iPAH patients improves exercise capacity and diminishes quadriceps muscle dysfunction by counteracting these structural muscle alterations. We therefore assessed the effects of an outpatient exercise training programme on exercise capacity, quadriceps function and quadriceps structure.

\section{METHODS}

\section{Study population}

In total, 19 patients were recruited from the VU University Medical Center (Amsterdam, the Netherlands) between 2006 and 2008 and met the following criteria: 1) diagnosed with iPAH according to World Health Organization criteria [19] established by right heart catheterisation; 2) stable clinical condition, defined as a change in 6-min walk distance (6MWD) of $<10 \%$ in three consecutive measurements prior to inclusion (over a period of minimally $1 \mathrm{yr}$ ), and no change in medical therapy for $\geqslant 3$ months; 3 ) aged 18 yrs or older; and 4 ) living within $5 \mathrm{~km}$ of a rehabilitation centre associated with the current study.

The Institutional Review Board on Research Involving Human Subjects (Amsterdam, the Netherlands) approved the protocol. Informed consent was obtained from all subjects.

\section{Study protocol}

Patients were evaluated at baseline and after 12 weeks, on two consecutive days. On day 1 a cardiopulmonary exercise test (CPET), quadriceps function tests and pulmonary function tests [20] were performed, and N-terminal pro-B-type natriuretic peptide (NT-proBNP) was determined. On day 2, an endurance exercise test was performed and the 6MWD was determined. 12 out of 19 patients underwent a quadriceps muscle biopsy on the second day of evaluation before and after training.

All patients attended an exercise training programme three times a week in a rehabilitation centre for a period of 12 weeks. The training programme was performed in rehabilitation centres nearby, according to usual clinical care. The standardised exercise protocol was adopted from the American Heart Association guidelines for rehabilitation of chronic heart failure patients [5]. The exercise training consisted of cycling and quadriceps muscle training (table 1). For safety reasons, physiotherapists, who recorded heart rate and oxygen saturation, always accompanied the patients. When oxygen saturation dropped $<85 \%$, or heart rate exceeded $120 \mathrm{bpm}$, the training session was paused or terminated early. Rehabilitation physicians or pulmonologists were on site and directly available for consultation. Attendance was recorded as an indicator for patient's compliance.

\section{Exercise testing}

6MWD test was performed according to American Thoracic Society guidelines [21]. Maximal capacity was determined during CPET as described previously [22]. During CPET heart frequency, pulse oximetry and gas exchange (breath-bybreath) were recorded. Anaerobic threshold was determined by the V-slope method [23].

Endurance capacity was evaluated by a submaximal exercise test performed at a constant load of $75 \%$ of baseline peak workload [24]. After $3 \mathrm{~min}$ of rest and $3 \mathrm{~min}$ of unloaded cycling, the patients had to exercise at $75 \%$ of baseline peak workload for as long as possible or the observer terminated the test after $15 \mathrm{~min}$. During the submaximal test heart frequency, pulse oximetry and gas exchange (breath by breath) were recorded.

\section{Quadriceps muscle function and biopsy}

Quadriceps function was assessed with a hydraulic dynamometer, as previously described [25]. In addition, 12 of 19 patients gave informed consent for a quadriceps muscle microbiopsy at baseline and after training, to analyse the effects of training on structural changes of the quadriceps muscle. Circumference of the leg was measured. Under local anesthetics ( $2 \%$ lidocaine), biopsies were taken from the vastus lateralis of the quadriceps muscle $10 \mathrm{~cm}$ above the patella with a 16G spring-loaded biopsy needle (QC-16-15.0-10T; Cook Medical, Limerick, Ireland). The biopsy was immediately evaluated under the microscope, embedded in $15 \%$ gelatin in Tyrode's solution containing $20 \mathrm{mM}$ butanedione monoxime and frozen in liquid nitrogen. Serial cryosections were cut at $-20^{\circ} \mathrm{C}$ and collected on slides coated with Vectabond (Vector Laboratories, Burlingame, CA, USA).

\section{Number of capillaries in quadriceps muscle}

Capillarisation of the quadriceps muscle was determined by quantitative immunofluorescence microscopy. Briefly, quadriceps cryosections $(5 \mu \mathrm{m})$ were incubated for $60 \mathrm{~min}$ with primary

\section{TABLE 1 12-week supervised exercise training protocol}

\begin{tabular}{|c|c|c|c|c|}
\hline Week & Intensity & Exercise & Rest time min & Sets \\
\hline \multicolumn{5}{|l|}{ Cycling } \\
\hline $1-3$ & $50 \% V^{\prime} \mathrm{O}_{2}, \max$ & $2 \min$ & 2 & 10 \\
\hline $4-6$ & $50 \% V^{\prime} \mathrm{O}_{2}, \max$ & $3 \mathrm{~min}$ & 2 & 7 \\
\hline $7-9$ & $75 \% V^{\prime} \mathrm{O}_{2}, \max$ & $4 \min$ & 2 & 6 \\
\hline $10-12$ & $75 \% V^{\prime} \mathrm{O}_{2}, \max$ & $5 \mathrm{~min}$ & 2 & 5 \\
\hline \multicolumn{5}{|c|}{ Quadriceps strength } \\
\hline $1-3$ & $50 \%$ ORM & 12 rep & 1 & 3 \\
\hline $4-6$ & $50 \%$ ORM & 13 rep & 1 & 3 \\
\hline $7-9$ & $75 \%$ ORM & 14 rep & 1 & 3 \\
\hline $10-12$ & $75 \%$ ORM & 15 rep & 1 & 3 \\
\hline \multicolumn{5}{|c|}{ Quadriceps endurance } \\
\hline $1-3$ & $30 \%$ ORM & 30 rep & 1 & 3 \\
\hline $4-6$ & $30 \%$ ORM & 40 rep & 1 & 4 \\
\hline $7-9$ & $40 \%$ ORM & 50 rep & 1 & 5 \\
\hline $10-12$ & $40 \%$ ORM & 60 rep & 1 & 6 \\
\hline
\end{tabular}

$V^{\prime} \mathrm{O}_{2}$,max: maximal oxygen uptake determined during cardiopulmonary exercise testing; ORM: one repetition maximum; rep: repetition. The exercise training consisted of cycle training (based on $\mathrm{V}^{\prime} \mathrm{O}_{2}$, max assessed at baseline measurements) and quadriceps training (based on ORM assessed on the first day of training). 
CD31-antibody (1:100; sc-1506-R; Santa Cruz Biotechnology, Santa Cruz, CA, USA), followed by secondary antibody staining with anti-rabbit Alexa fluor 488 (Vector Laboratories), as well as wheat germ agglutinin (glycocalyx; Molecular Probes, Invitrogen, Paisley, UK) and 4',6'-diamidino-2-phenylindole (nuclei; Molecular Probes, Invitrogen) counterstaining. Image acquisition was performed on a Marianas digital imaging microscopy workstation (Intelligent Imaging Innovations (3i), Denver, CO, USA) using multiple fluorescence channels. SlideBook imaging analysis software (SlideBook 4.2 ; 3i) was used to semi-automatically quantify the images. Capillarisation was expressed as the number of capillaries per quadriceps myocyte measured in the whole section.

\section{Fibre type distribution, oxidative enzyme activity and cross- sectional area}

Cross-sectional area (CSA), fibre type distribution and oxidative enzyme activity were determined as previously described $[26,27]$. In detail, in 10 randomly selected type I and 20 randomly selected type II cells (identified by serial sections stained for myofibrillar ATPase), oxidative enzyme activity was analysed by measuring succinate dehydrogenase (SDH) absorbance at $660 \mathrm{~nm}(10 \mu \mathrm{m}$ sections incubated for $20 \mathrm{~min}$ at $37^{\circ} \mathrm{C}$ ). For the same cells, CSA was measured. Fibre type distribution was analysed by counting all type I and type II muscle fibres in the biopsy. Images were analysed using ImageJ imaging analysis software (ImageJ for Windows 1.39a; $\mathrm{NIH}$, Bethesda, MD, USA).

\section{Statistics}

All data are presented as mean \pm SEM, unless stated otherwise. A p-value $<0.05$ was considered statistically significant. The effects of training on exercise capacity, quadriceps function and quadriceps capillarisation were assessed by a paired t-test. Changes in fibre type distribution were assessed by two-way repeated measures ANOVA. Oxidative enzyme activity and muscle fibre CSA were analysed by multi-level analyses, to correct for the nonindependence of successive measurements per patient [28]. Regression analyses were performed to study the association between changes in quadriceps endurance and aerobic capacity. Analyses were performed with SPSS 16.0 (SPSS Inc., Chicago, IL, USA) or MLwiN 2.02 software (University of Bristol, Bristol, UK).

\section{RESULTS}

In total, 19 patients were recruited for the present study and their baseline characteristics are presented in table 2 . The patient cohort was typical iPAH, with a female predominance, median New York Heart Association class III, and a mean age of 42 yrs. In addition, the last known catheterisation data $(<1 \mathrm{yr})$ prior to the rehabilitation are given in table 2 .

The training programme was well tolerated by all patients and no adverse events were observed. The compliance to the exercise training programme was $91 \pm 2 \%$. Only two patients required a minor training adjustment, because of complaints of dizziness during the quadriceps exercise. Training did not elevate NT-proBNP levels $\left(947 \pm 429\right.$ to $1,043 \pm 462 \mathrm{pg} \cdot \mathrm{mL}^{-1}$; nonsignificant) and did not change pulmonary function (data not shown).

\section{Exercise training improved endurance}

The CPET revealed no significant improvements of maximal capacity and gas exchange after training. Moreover, exercise training did not improve 6MWD (fig. 1a). However, endurance exercise characteristics improved significantly after training, demonstrated by a shift of anaerobic threshold to a higher workload (from $32 \pm 5$ to $46 \pm 6 \mathrm{~W} ; \mathrm{p}=0.003$ ). In addition, exercise endurance time increased by $89 \%$ after training (fig. $1 b ; \mathrm{p}<0.001$ ).

\section{Exercise training improved quadriceps function and aerobic capacity}

Quadriceps muscle strength improved modestly by $13 \%$ after training (fig. 1c; from $94 \pm 7$ to $106 \pm 8 \mathrm{Nm}$; $\mathrm{p}=0.005$ ). Quadriceps endurance improved markedly by 34\% after training (fig. 1d; from $136 \pm 10$ to $181 \pm 18 \mathrm{~s} ; \mathrm{p}=0.001$ ).

Leg circumference did not change after training (from $43 \pm 1$ to $44 \pm 1 \mathrm{~cm}$ ). No increase in CSA was found in both type I (from

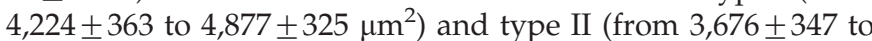
$4,235 \pm 462 \mu \mathrm{m}^{2}$ ) muscle fibres (fig. 2a). Fibre type distribution did not change after training (fig. $2 b$; type I from $35 \pm 3$ to $38 \pm 3 \%$; type II from $65 \pm 3$ to $62 \pm 3 \%$ ).

However, training increased the number of capillaries per myocyte by $30 \%$ (fig. 3; from $1.36 \pm 0.10$ to $1.78 \pm 0.13$; $\mathrm{p}<0.001$ ). SDH absorbance increased by $39 \%$ in type I (slow) muscle fibres (fig. 4 ; from $0.161 \pm 0.011$ to $0.216 \pm 0.020$ $\mathrm{p}<0.001$ ) whereas it increased by $30 \%$ in type II (fast) muscle fibres (from $0.105 \pm 0.009$ to $0.133 \pm 0.010 ; p=0.05$ ).

\begin{tabular}{|c|c|}
\hline Subjects & 19 \\
\hline Male/female & $4 / 15$ \\
\hline Age yrs & $42 \pm 13$ \\
\hline Height m & $1.70 \pm 0.09$ \\
\hline Weight kg & $72 \pm 14$ \\
\hline NYHA class II/III & $3 / 16$ \\
\hline NT-proBNP pg.mL ${ }^{-1}$ & $947 \pm 1606$ \\
\hline \multicolumn{2}{|l|}{ Haemodynamics } \\
\hline $\bar{P}$ pa mmHg & $53 \pm 19$ \\
\hline PVR dyn $\cdot s \cdot \mathrm{cm}^{-5}$ & $845 \pm 460$ \\
\hline Cardiac index $\mathrm{L} \cdot \mathrm{min}^{-1} \cdot \mathrm{m}^{-2}$ & $2.7 \pm 0.9$ \\
\hline Right atrial pressure $\mathrm{mmHg}$ & $7 \pm 4$ \\
\hline $\bar{P}$ pcw mmHg & $5.7 \pm 2.4$ \\
\hline \multicolumn{2}{|l|}{ Exercise capacity } \\
\hline 6-min walk distance m & $496 \pm 108$ \\
\hline Peak work W & $78 \pm 42$ \\
\hline Peak $V^{\prime} \mathrm{O}_{2} \mathrm{~mL} \cdot \mathrm{kg}^{-1} \cdot \mathrm{min}^{-1}$ & $15 \pm 4$ \\
\hline Peak $V^{\prime} E L \cdot \min ^{-1}$ & $63 \pm 30$ \\
\hline Peak $V^{\prime} E / V^{\prime} \mathrm{CO}_{2}$ & $51 \pm 13$ \\
\hline \multicolumn{2}{|l|}{ Medication } \\
\hline Single/combination treatment & $8 / 11$ \\
\hline Treatment duration months & $20 \pm 16$ \\
\hline
\end{tabular}

Data are presented as $n$ or mean \pm SD. NYHA: New York Heart Association; NTproBNP: N-terminal pro-B-type natriuretic peptide; $\bar{P}$ pa: mean pulmonary artery pressure; PVR: pulmonary vascular resistance; $\bar{P}_{\text {pcw: }}$ pulmonary capillary wedge pressure; $V^{\prime} \mathrm{O}_{2}$ : oxygen uptake; $V^{\prime} E$ : minute ventilation; $V^{\prime} E / V^{\prime} \mathrm{CO}_{2}$ ventilatory equivalent for carbon dioxide. 

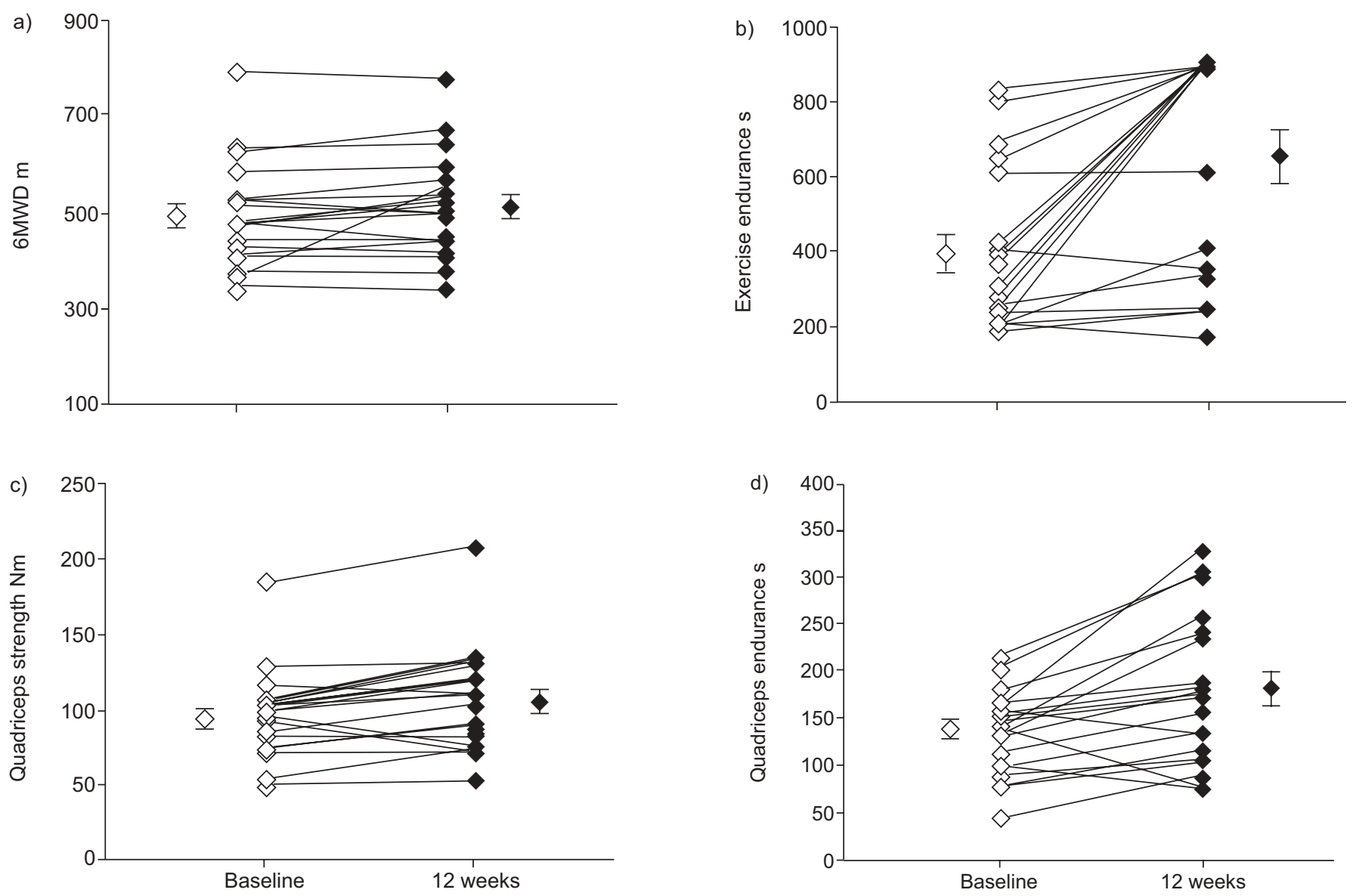

FIGURE 1. Effect of a 12-week exercise training programme on a) 6-min walk distance (6MWD), b) exercise endurance, c) quadriceps strength and d) quadriceps endurance. Data are presented as mean \pm SEM. $\diamond$ : baseline values;

: values after 12 weeks of training individual patients.

The change in SDH absorbance of the type I (slow) muscle fibres and the change in the number of capillaries were highly associated with the improvements of quadriceps endurance $\left(\mathrm{r}^{2}=0.73 ; \mathrm{p}<0.001\right)$.

\section{DISCUSSION}

To the best of our knowledge, this is the first study that evaluates the effect of exercise training on quadriceps function

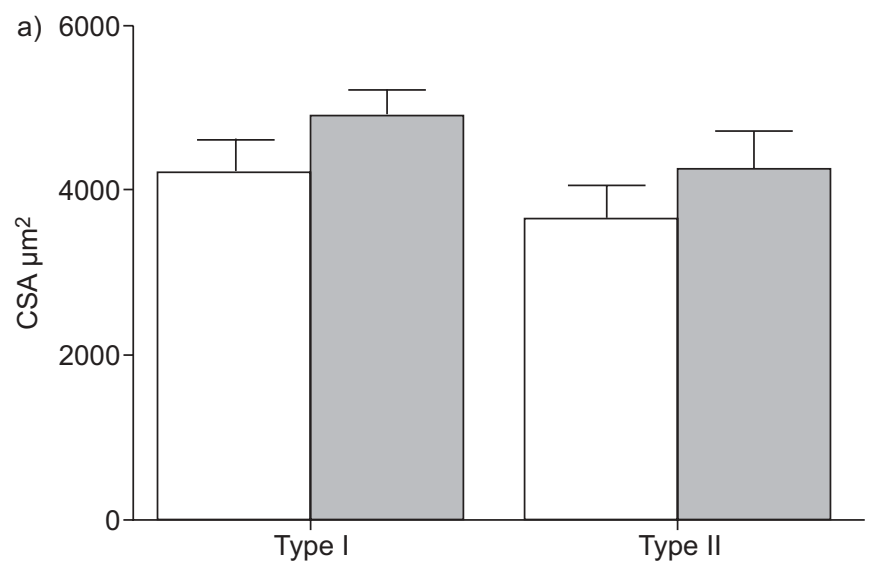

and morphology in iPAH patients. We have demonstrated that a 12-week outpatient training protocol increases endurance capacity, without an improvement of maximal capacity. The same phenomenon was seen on quadriceps function: a large improvement in quadriceps endurance was found, and a small (but significant) increase in quadriceps strength. Histological analyses revealed improved aerobic capacity by increased quadriceps capillarisation and oxidative enzyme activity,

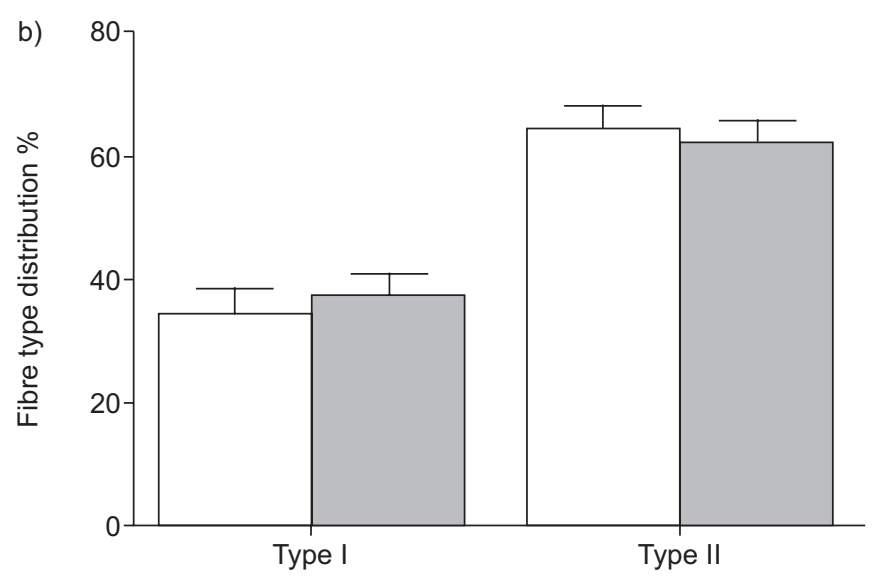

FIGURE 2. a) Cross-sectional area (CSA) and b) fibre type distribution after 12 weeks of training in both type I (slow) and type II (fast) muscle fibres. Data are presented as mean \pm SEM. $\square$ : baseline values; $\square$ : values after training. 

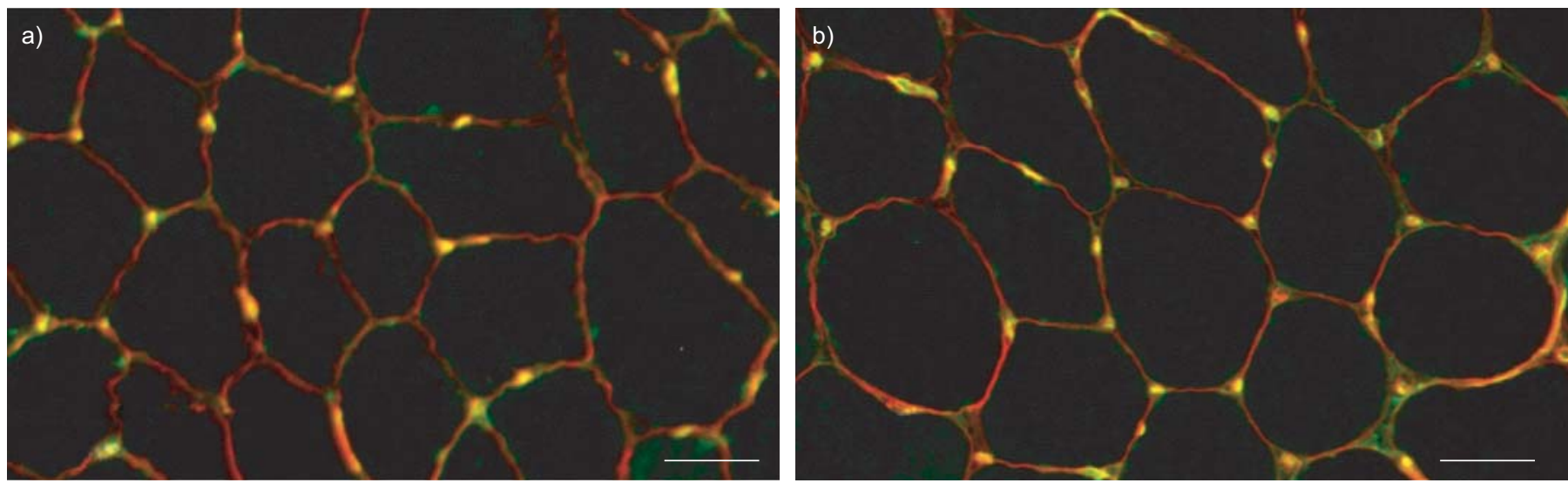

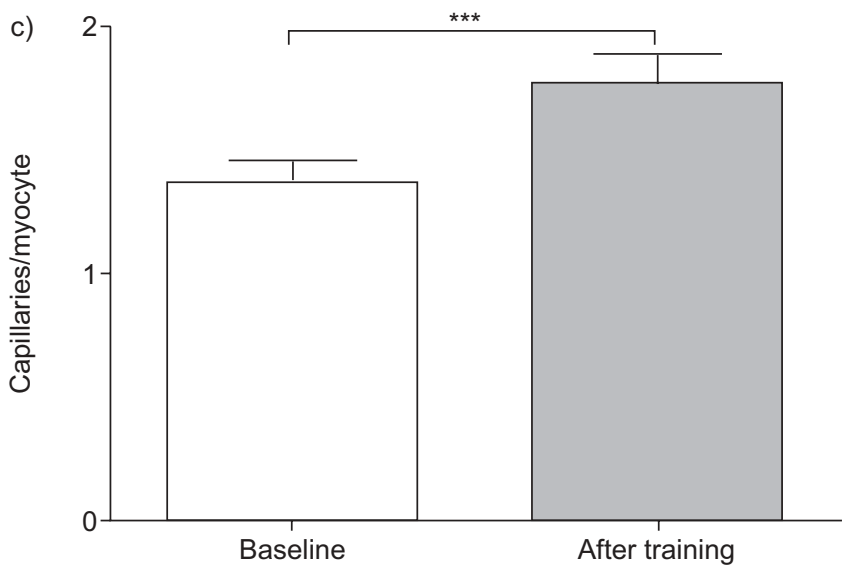

without hypertrophy or fibre type switch. Finally, these morphological changes were strongly correlated with an improvement in quadriceps endurance.

\section{Training improved endurance capacity}

In the first clinical trial by MERELES et al. [4], investigating the effect of training in pulmonary hypertension, a major improvement was found in 6MWD, which was even greater than any medical intervention had achieved previously. They also reported improvements in both maximal as well as submaximal exercise capacity. However, our study shows improvement only in endurance capacity. This can be explained by differences in training modalities and study population. First, our training schedule consisted of cycle and quadriceps training, whereas the training schedule of MERELES et al. [4] consisted of cycling and walking training. Furthermore, our training protocol was mainly focused on improving endurance capacity, which might explain the absence of improved maximal capacity. Secondly, baseline 6MWD of our patient population were slightly higher $(496 \pm 108 \mathrm{~m}$ in our study versus $439 \pm 82 \mathrm{~m}$ in the study of MERELES et al. [4]) which may imply that our patients had a smaller window of improvement in 6MWD. In addition to the Mereles et al. [4] study, we were able to confirm the improvement of endurance capacity with an endurance exercise test, where we found an improvement in endurance time of $89 \%$. Moreover, we observed the same phenomenon in quadriceps function; quadriceps strength was only modestly
FIGURE 3. Typical examples of quadriceps capillarisation in one patient a) before and b) after training (red: cell membrane of the quadriceps myocytes: yellow: capillaries). c) Number of capillaries per quadriceps myocyte at baseline and after training. Data are presented as mean \pm SEM. Scale bars $=50 \mu \mathrm{m}$. ***: $\mathrm{p}<0.001$.

improved, whereas quadriceps endurance improved significantly by $34 \%$.

Recently, BOUTET et al. [29] presented preliminary results from their 12-week outpatient rehabilitation programme in patients with iPAH. Interestingly, they also only found improvements in endurance but not in maximal capacity, which is in line with our findings.

\section{Improved quadriceps endurance is associated with increased aerobic capacity}

Quadriceps dysfunction is often observed in several chronic diseases such as COPD and chronic heart failure. More recently, several reports suggested muscle dysfunction in patients with pulmonary arterial hypertension [14-16]. Mechanisms are still unclear, but it can be speculated that inactivity of the skeletal muscles, together with a decreased cardiac output leading to a reduced oxygen transport to the skeletal muscles, trigger morphological changes, such as muscle atrophy, fibre type switching and reduced aerobic capacity [18].

In patients with COPD or heart failure, prolonged exercise training is an effective tool to reverse changes in mitochondria, key metabolic enzymes, capillarisation and, to a lesser extent, causes changes in fibre type composition [13]. These findings are in line with our findings of improved oxidative enzyme activity and number of capillaries. However, we did not find changes in CSA or fibre type distribution, which might be a 

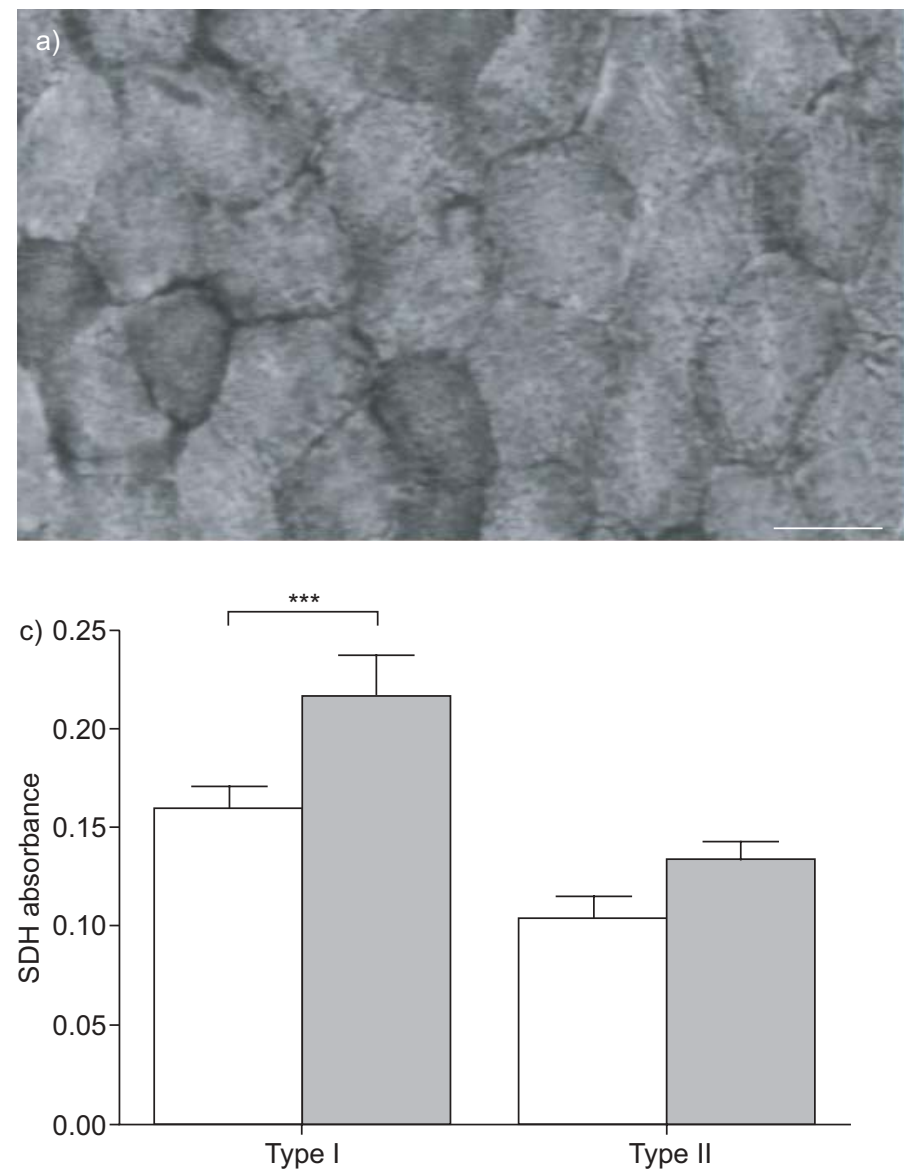

consequence of the relative short exercise period at a low intensity [12].

\section{Limitations}

To reduce the burden of the present study for the patients, we limited the number of tests and the invasiveness of the measurements. We were therefore not able to assess the effect of training on quadriceps muscle function with electrical stimulation. A potential bias due to differences in motivation before and after training, may have overestimated the observed improvements in quadriceps function. However, as we found a strong association between quadriceps function and biopsy data, motivation differences may have had only a minor effect on outcome. Moreover, to limit the number of measurements, we did not measure quadriceps muscle mass by magnetic resonance imaging. Based on the measurements of leg circumference and CSA of the individual muscle fibres, changes in muscle mass are unlikely. To decrease the invasiveness of a muscle biopsy, we used a microbiopsy technique instead of the Bergström method [30, 31]. As a consequence, protein analyses were not performed. However, we used well validated histological techniques. For instance, capillary density was semi-automatically quantified with a CD31 antibody, which is a standard method when investigating angiogenesis $[9,32]$.

\section{Clinical relevance}

The current study confirms that iPAH is not only associated with compromised cardiopulmonary function but also with

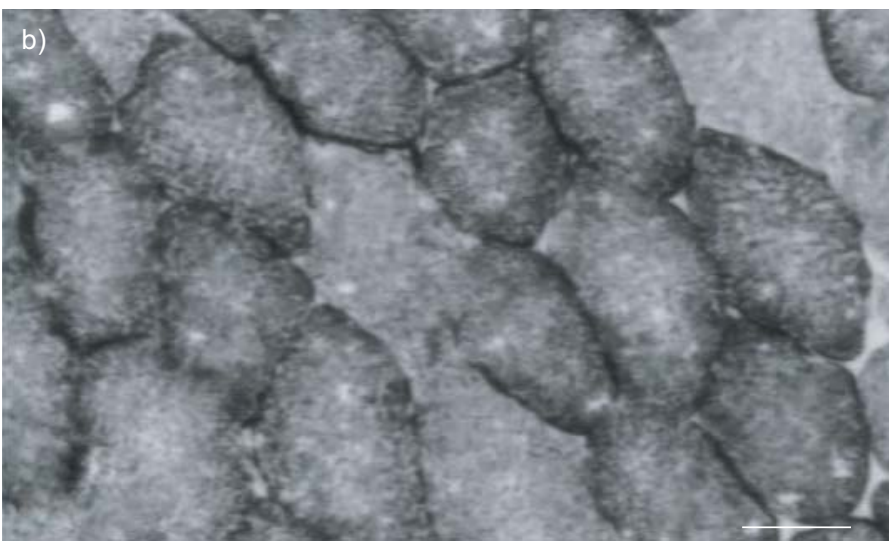

FIGURE 4. Typical examples of oxidative enzyme activity in one patient a) before and b) after training (dark cells indicate high oxidative enzyme activity, light cells indicate low oxidative enzyme activity). c) Training increased oxidative enzyme activity especially in the type I (slow) muscle fibres. Data are presented as mean \pm SEM; $\square$ : baseline values; $\square$ : values after training. SDH: succinate dehydrogenase. Scale bars $=50 \mu \mathrm{m}$. ${ }^{* *}: \mathrm{p}<0.001$.

impaired skeletal muscle function. We found that skeletal muscle dysfunction could partially be reversed by exercise training in iPAH. Moreover, our training programme improved quadriceps endurance, more than quadriceps strength, even though both aspects were implemented in the training protocol. This implies that future training protocols should focus on enhancing endurance capacity rather than maximal capacity.

Although we have found an overall beneficial effect of exercise training, we cannot yet generally recommend exercise training for all PAH patients. For instance, we were not able to rule out repercussions on cardiac function and haemodynamics, although after training NT-proBNP levels remained unaltered. Moreover, not all patients seemed to benefit from the exercise training therapy (fig. 1b). However, the relatively small number of patients did not allow us to discriminate responders from nonresponders at baseline. Future studies and clinical trials should asses the effects of training on right ventricular remodelling and function, and determine factors that can predict which patients can benefit most from exercise training.

\section{Conclusion}

Exercise training improves endurance and quadriceps muscle function, which is also reflected by structural changes in the quadriceps muscle. Our present study supports the potential role of exercise training as an adjunct therapy in stable iPAH patients. 


\section{STATEMENT OF INTEREST}

None declared.

\section{ACKNOWLEDGEMENTS}

The present authors would like to thank all patients who participated in the current study and the physiotherapists who supervised the training sessions.

\section{REFERENCES}

1 Deboeck G, Niset G, Lamotte M, et al. Exercise testing in pulmonary arterial hypertension and in chronic heart failure. Eur Respir J 2004; 23: 747-751.

2 Yasunobu Y, Oudiz RJ, Sun XG, et al. End-tidal $P_{C_{2}}$ abnormality and exercise limitation in patients with primary pulmonary hypertension. Chest 2005; 127: 1637-1646.

3 Desai SA, Channick RN. Exercise in patients with pulmonary arterial hypertension. J Cardiopulm Rehabil Prev 2008; 28: 12-16.

4 Mereles D, Ehlken N, Kreuscher S, et al. Exercise and respiratory training improve exercise capacity and quality of life in patients with severe chronic pulmonary hypertension. Circulation 2006; 114: 1482-1489.

5 Pollock ML, Franklin BA, Balady GJ, et al. AHA Science Advisory. Resistance exercise in individuals with and without cardiovascular disease: benefits, rationale, safety, and prescription. An advisory from the Committee on Exercise, Rehabilitation, and Prevention, Council on Clinical Cardiology, American Heart Association; Position paper endorsed by the American College of Sports Medicine. Circulation 2000; 101: 828-833.

6 Nici L, Donner C, Wouters E, et al. American Thoracic Society/ European Respiratory Society statement on pulmonary rehabilitation. Am J Respir Crit Care Med 2006; 173: 1390-1413.

7 Clark AL, Poole-Wilson PA, Coats AJ. Exercise limitation in chronic heart failure: central role of the periphery. J Am Coll Cardiol 1996; 28: 1092-1102.

8 Gosselink R, Troosters T, Decramer M. Peripheral muscle weakness contributes to exercise limitation in COPD. Am J Respir Crit Care Med 1996; 153: 976-980.

9 Duscha BD, Kraus WE, Keteyian SJ, et al. Capillary density of skeletal muscle: a contributing mechanism for exercise intolerance in class II-III chronic heart failure independent of other peripheral alterations. J Am Coll Cardiol 1999; 33: 1956-1963.

10 Ventura-Clapier R, Mettauer B, Bigard X. Beneficial effects of endurance training on cardiac and skeletal muscle energy metabolism in heart failure. Cardiovasc Res 2007; 73: 10-18.

11 Maltais F, Leblanc P, Simard C, et al. Skeletal muscle adaptation to endurance training in patients with chronic obstructive pulmonary disease. Am J Respir Crit Care Med 1996; 154: 442-447.

12 Hambrecht R, Fiehn E, Yu J, et al. Effects of endurance training on mitochondrial ultrastructure and fiber type distribution in skeletal muscle of patients with stable chronic heart failure. J Am Coll Cardiol 1997; 29: 1067-1073.

13 Lampert E, Mettauer B, Hoppeler H, et al. Skeletal muscle response to short endurance training in heart transplant recipients. J Am Coll Cardiol 1998; 32: 420-426.
14 Meyer FJ, Lossnitzer D, Kristen AV, et al. Respiratory muscle dysfunction in idiopathic pulmonary arterial hypertension. Eur Respir J 2005; 25: 125-130.

15 Kabitz HJ, Schwoerer A, Bremer HC, et al. Impairment of respiratory muscle function in pulmonary hypertension. Clin Sci (Lond) 2008; 114: 165-171.

16 Bauer R, Dehnert C, Schoene P, et al. Skeletal muscle dysfunction in patients with idiopathic pulmonary arterial hypertension. Respir Med 2007; 101: 2366-2369.

17 de Man FS, van 't Hul AJ, van der Laarse WJ, et al. Exercise training in pulmonary arterial hypertension. Eur Respir J 2006; 28 : Suppl. 50, 641s.

18 Naeije R. Breathing more with weaker respiratory muscles in pulmonary arterial hypertension. Eur Respir J 2005; 25: 6-8.

19 Simonneau G, Galie N, Rubin LJ, et al. Clinical classification of pulmonary hypertension. J Am Coll Cardiol 2004; 43: 5S-12S.

20 Brusasco V, Crapo R, Viegi G. Coming together: the ATS/ERS consensus on clinical pulmonary function testing. Eur Respir J 2005; 26: 1-2.

21 American Thoracic Society, ATS statement: guidelines for the sixminute walk test. Am J Respir Crit Care Med 2002; 166: 111-117.

22 Holverda S, Bogaard HJ, Groepenhoff H, et al. Cardiopulmonary exercise test characteristics in patients with chronic obstructive pulmonary disease and associated pulmonary hypertension. Respiration 2008; 76: 160-167.

23 Wasserman K, Hansen JE, Sue DY, et al. Principles of Exercise Testing and Interpretation. Baltimore, Lippincott Williams \& Wilkins, 1999.

24 van 't Hul A, Gosselink R, Kwakkel G. Constant-load cycle endurance performance: test-retest reliability and validity in patients with COPD. J Cardiopulm Rehabil 2003; 23: 143-150.

25 van 't Hul A, Harlaar J, Gosselink R, et al. Quadriceps muscle endurance in patients with chronic obstructive pulmonary disease. Muscle Nerve 2004; 29: 267-274.

26 van der Laarse WJ, Diegenbach PC, Maslam S. Quantitative histochemistry of three mouse hind-limb muscles: the relationship between calcium-stimulated myofibrillar ATPase and succinate dehydrogenase activities. Histochem J 1984; 16: 529-541.

27 Bekedam MA, van Beek-Harmsen BJ, Boonstra A, et al. Maximum rate of oxygen consumption related to succinate dehydrogenase activity in skeletal muscle fibres of chronic heart failure patients and controls. Clin Physiol Funct Imaging 2003; 23: 337-343.

28 Twisk JWR. Applied multilevel analysis. Cambridge, University Press, 2006.

29 Boutet K, Garcia G, Degano B, et al. Results of a 12-week outpatient cardiovascular rehabilitation in patients with idiopathic pulmonary arterial hypertension. Eur Respir J 2008; 32: Suppl. 52, 240s.

30 Hayot M, Michaud A, Koechlin C, et al. Skeletal muscle microbiopsy: a validation study of a minimally invasive technique. Eur Respir J 2005; 25: 431-440.

31 Bergstrom J. Percutaneous needle biopsy of skeletal muscle in physiological and clinical research. Scand J Clin Lab Invest 1975; 35: 609-616.

32 Oyama O, Sugimoto N, Qi X, et al. The lysophospholipid mediator sphingosine-1-phosphate promotes angiogenesis in vivo in ischaemic hindlimbs of mice. Cardiovasc Res 2008; 78: 301-307. 